fracture in the normal population. The overall prevalence of identified risk factors was low but smoking and body weight are both modifiable thus health promotion messages should aim to target these risk factors.

\section{SAT0175 EFFECTS OF COMBINED TREATMENT ALENDRONATE + ALFACALCIDOL ON BONE MINERAL DENSITY IN POSTMENOPAUSAL OSTEOPOROSIS}

${ }^{1} \mathrm{~K}$ Önes, 'S Tetik, ${ }^{2} \mathrm{M}$ Eryavuz, ${ }^{2} \mathrm{U}$ Akarirmak, ${ }^{1} \mathrm{~N}$ Caglar, ${ }^{3} \mathrm{H}$ Yilmaz. ${ }^{1}$ Vakif Gureba Education Hospital, Physical Medicine and Rehabilitation Clinic; ${ }^{2}$ Department of PM\&R, Cerrahpasa Faculty of Medicine, Instanbul University; ${ }^{3}$ Research Unit, 70. Yil Rehabilitation Center, Istanbul, Turkey

\subsection{6/annrheumdis-2001.662}

\section{Background}

Objectives The purpose of this study was to evaluate the effect of four different treatment modalities on bone mineral density (BMD) in postmenopausal osteoporosis.

Methods 170 postmenopausal women BMD (measured by dual energy X-Ray absorptiometry-DXA) $\mathrm{T}$ scores at least ?2,5 SD below the peak young adult mean were included in the study. The patients were randomised to four groups. 40 patients were treated with alendronate $(10 \mathrm{mg} /$ daily $)+$ alfacalcidol $(0,5 \mu \mathrm{g} /$ daily) and 40 patients were treated alendronate $(10 \mathrm{mg} /$ daily $)$ and 60 patients were treated alfalcidol $(0,5 \mu \mathrm{g} /$ daily $)$ for two years. All patients received $500 \mathrm{mg}$ /daily supplemental calcium to ensure adequate calcium intake. Only $500 \mathrm{mg}$ of calcium were given to the control group of 30 patients. BMD was measured by total body dual-energy X-Ray absorptiometry (DPXLunar) in the lumbar spine and in proximal segments of the femur (neck, ward?s triangle, trochanter, inter and total hip). BMD was evalvated at baseline and after 12 and 24 months. The patients followed up for two years.

Results After two years of treatment, we found significant increase of femoral neck, trochanter, ward?s triangle and lumbar spine density in alendronate + alfacalcidol group and alone alendronate group $(\mathrm{p}<0.05)$. Also, in the second year of treatment, the combination of alendronate + alfacalcidol showed BMD increases that were significantly higher than for alendronate $(\mathrm{p}<0.05)$. In the alfacalcidol group, BMD no increased significantly $(\mathrm{p}>0.05)$.

In the placebo group (only calcium $500 \mathrm{mg} /$ daily), BMD decreased significantly $(\mathrm{p}<0.05)$.

No adverse effect were observed during the two years of the study, in particular with respect to renal function and the gastrointestinal system.

Conclusion These results showed that combined treatment with alfacalcidol + alendronate was more effective than therapy with alendronate alone.

\section{SAT0176 THYROID AND BONE METABOLISM}

${ }^{1} \mathrm{M}$ Bernardes, ${ }^{1} \mathrm{~A}$ Bernardo, ${ }^{1} \mathrm{C}$ Vaz, ${ }^{2} \mathrm{M}$ Ramires, ${ }^{1} \mathrm{E}$ Mariz, ${ }^{1} \mathrm{~F}$ Brandão, ${ }^{1} \mathrm{P}$ Valente, ${ }^{1} \mathrm{~A}$ Lopes-Vaz. 'Rheumatology Department; ${ }^{2}$ Endocrinology Department, São João Hospital, Porto, Portugal

\subsection{6/annrheumdis-2001.663}

Background Hyperthyroidism (HT) is a risk factor for osteoporosis (OP). It is still uncertain if bone mass and bone turnover are completely normalised after treatment of HT.
Objectives The aim of the present cross-sectional study was to investigate whether former hyperthyroid patients have an increased risk for OP due to reduced bone mass and/or persisting high bone remodelling activity after euthyroidism was restored.

Methods We studied 53 formerly hyperthyroid females (age39 \pm 13 years;20 premenopausal (premw); 33 postmenopausal (posmw), who had been euthyroid for a mean period $129 \pm 78$ months. They were treated by combined medical therapy.we evaluated bone mineral density (BMD) with Lunar4500 DEXA and calcaneal quantitative ultrasound (QUS) parameters with Osteometer DTUone. Biochemical markers of bone turnover (serum osteocalcin (OC), beta-crosslaps (BCL) and urinary deoxypiridinoline (U-DPR) and cross-linked N-telopeptides of type I collagen (NTX), thyroid hormones and antithyroid antibodies (atb) were measured.

Results Considering DEXA parameters, in posmw,18\% had vertebral OP and 7\%had hip OP. A higher prevalence of OP was detected by QUS evaluation:39\%BUA tscore $\ll-2,5 \mathrm{sd}$ and $42 \%$ SOStscore $\ll-2,5 \mathrm{sd}$. There were no cases of OP in premw. Abnormally high levels of U-NTX (35\%premw, 61\%posmw), U-DPR (26\% premw, $15 \%$ posmw) and BCL (26\% premw, $52 \%$ posmw) were found. Significant correlations were detected between UNTX and TSH $(\mathrm{p}<0,05, \mathrm{r}=-0,45)$, U-NTX and free T3 in premw $(\mathrm{p}<0,05, \mathrm{r}=0,47), \mathrm{BCL}$ and antithyroidperoxidase atb ( $\mathrm{p}<0,05, \mathrm{r}=0,56$ ), BCL and antithyroglobulin atb in posmw $(\mathrm{p}=0,001, \mathrm{r}=0,59)$, OC and antithyroglobulin atb in posmw $(\mathrm{p}=0,001, \mathrm{r}=0,60)$ and $\mathrm{OC}$ and antithyroidperoxidase atb in posmw ( $\mathrm{p}<0,001, \mathrm{r}=0,64)$. There was no correlation between these biomarkers and dose or duration of thyroxin replacement therapy.

Conclusion The prevalence of OP in former hyperthyroid women wasn 't superior to the general population. There was no significant difference of $\mathrm{BMD}($ Zscores $«-2,5=0)$, although these women presented increased bone resorption markers. The discrepancy between DEXA and QUS could reflect differences in "bone quality". Curiously, dispite being euthyroid, the correlation between U-NTX and TSH could signify that TSH levels close to inferior limit of normality are associated with an increased bone turnover. There is also no explanation to the correlation between biomarkers and antithyroid atbs, but we can ${ }^{\prime} t$ exclude a possible role of this atbs in the production of BCL or OC.

\section{REFERENCES}

1 Langdahl, et al. Bone mass, bone turnover, body composition and calcium homeostasis in former hyperthyroid patients treated by combined medical therapy. Thyroid 1996;6(3):161-8

2 Grant, et al. Is previous hyperthyroidism still a risk factor for osteoporosis in postmenopausal women? Clin Endocrinol. 1995;43:339-45

\section{SAT0177 GENDER, AGE AND ANTHROPOMETRIC CORRELATIONS OF HIP AXIS LENGTH}

${ }^{1} \mathrm{M}$ Salvador, ${ }^{1} \mathrm{CA}$ Silva, ${ }^{2} \mathrm{~V}$ Rodrigues, ${ }^{3} \mathrm{JA}$ Da Silva. ${ }^{1}$ Department of Rheumatology; ${ }^{2}$ Department of Epidemiology, Faculty of Medicine, Coimbra, Portugal; ${ }^{3}$ Rheumatology Clinic of Coimbra, University Hospital of Coimbra

\subsection{6/annrheumdis-2001.664}

Background Hip axis length (HAL) has been proposed as an important risk factor for hip fracture, independent of bone mineral density. Significant differences of HAL have been demonstrated in relation to gender, age and ethnic origin. The latter 